\title{
Abstracts \\ I International Symposium on Animal Models for Translational Research: Prostate Cancer
}

\author{
Submitted March $13^{\text {th }}$ 2018, Accepted May $10^{\text {th }} 2018$
}

Financial Support: Fapesp

Cross species analysis of syndecan proteoglycans expression in prostate cancer

Nilton J. Santos*1, Caroline N. Barquilha' ${ }^{1}$, Isabela C. Barbosa $^{2}$, Isabela G. F. Paiva², Sérgio Luis Felisbino ${ }^{2}$

${ }^{1}$ Institute of Biology (IB) - University of Campinas (UNICAMP), Campinas/SP, Brazil.

${ }^{2}$ Institute of Biosciences of Botucatu (IBB) - Sao Paulo State University (UNESP), Botucatu/SP, Brazil.

*Corresponding author: njsantos@outlook.com.br

Background: Prostate cancer (PCa) is the second most frequent among men. Studies highlight the role of cell surface proteoglycans, such as the syndecan family (SDC) in initiation and progression of CaP. The study aimed to characterize the gene expression pattern and tissue localization of the proteoglycans of the SDC family 1-4 and its Syntenin-1 binding protein. Methodology: A meta-analysis of the gene expression of these 5 genes was performed in the different normal and tumor prostate lobes of the knockout mice for the Pten gene (Pten model) and $\mathrm{Tp} 53$ and Rb1 (p53/Rb) genes, GEO dataset (GSE94574). Formalin-paraffin processed tumor samples of the two knockout models and human prostate were submitted to immunohistochemistry for Sindecan 1-4 (SDCs) and Sintenin-1. Results and Discussion: Gene expression analysis showed Pten mouse tumors presented significant increase in the expression of SDC1 and SDC4, while $\mathrm{p} 53 / \mathrm{Rb}$ model, there were an expressive increase in SDC3 expression and a significant reduction in the expression of SDC2 and SDC4. In human prostates cancer, SDCs and syntenin showed variable expression in epithelial and stromal cells, with tumors being positive and others negative, but a correlation with poor prognosis and lower survival rate was observed for Syndecan 2 and 3 . Conclusions: Our results suggest that the expression of SDC1 and SDC4 may be related to indolent tumors and in patients with better prognosis. SDC2 and, especially,
SDC3 may be involved in more aggressive tumors, such as neuroendocrine tumors, and worse prognosis for patients.

\section{Effects of hormonal imbalance on stromal compartment of Gerbil prostate}

Julia Q. Antoniassi ${ }^{1 *}$, Sarah B. Augusto ${ }^{2}$, Luiz Henrique A. Guerra $^{3}$, Ricardo Alexandre Fochi ${ }^{3}$, Luis Roberto F. Junior $^{4}$, Sebastião R. Taboga ${ }^{4}$

${ }^{1}$ Department of Structural and Functional Biology, State University of Campinas, Campinas, São Paulo, Brazil.

${ }^{2}$ Cancer Hospital of Barretos, Barretos, São Paulo, Brazil.

${ }^{3}$ University Center of Rio Preto, São José do Rio Preto, São Paulo, Brazil.

${ }^{4}$ Department of Biology, São Paulo State University, São José do Rio Preto, São Paulo, Brazil.

*Corresponding author: julia.antoniassi@gmail.com

The stromal compartment responds quickly to adverse situations, such as injury repair and response to disorders in tissue homeostasis, which may also result from the genesis of cancer. In this way, the components of the stromal microenvironment can act when the epithelium is damaged. This study aimed elucidate the effects of hormonal imbalance, in the stromal compartment, caused by inhibition of $5 \alpha$-redutase and Aromatase. Adult gerbils $(\mathrm{n}=5) \quad$ received $\quad$ Finasteride (F - $10 \mathrm{mg} / \mathrm{Kg} /$ day, $5 \alpha$-redutase inhibitor) or Chrysin $(\mathrm{Cr}-$ $50 \mathrm{mg} / \mathrm{Kg} /$ day, Aromatase inhibitor) for 4 weeks. The prostate was fixed and processed for general and immunostaining ( $\alpha$-actin, TGF- $\beta$, MMP-9, MMP- 2 and CD34) analyses, and the hormonal levels were determined by ELISA. The inhibitors caused a reduction of testosterone levels and, in group F, there was an increase of estradiol. Both treatments caused similar morphological changes, such as: presence of cell debris inside the lumen, accumulation of collagen in the subepithelial layer, 
reduction of secretion, presence of inflammatory foci and rupture of the muscular layer around the acini. It was observed $\mathrm{In} \mathrm{Cr}$ a secretion with spiny aspects. Clear cells and epithelial cells with accumulation of secretory vesicles was found in $F$. The treatments led to changes in the pattern of different immunoblotting, with increased labeling of TGF $\beta$ and MMP9. In addition, MMP2 increased in $\mathrm{F}$. The percentage of $\mathrm{CD} 34+$ cells also increased. The results showed that the treatment period is insufficient for complete inhibition of the glands, despite this they triggered important prostatic alterations.
Financial
support:
FAPESP
$(2014 / 26660$

$0) / \mathrm{CAPES} / \mathrm{CNPq}$.

CEUA/Ibilce-UNESP/Protocol: 130/2016

\section{Effects of Leishmaniasis caused by Leishmania (Viannia) braziliensis on Hamster Ventral Prostate}

Mônica O. Teles ${ }^{1 *}$, Camila Carolini F. Marques ${ }^{1}$, Gessilda A. Nogueira-Melo ${ }^{2}$, Jaqueline C. Rinaldi ${ }^{2}$

${ }^{1}$ Undergraduated.

${ }^{2} \mathrm{PhD}$ - State University of Maringa - UEM, Maringá/PR.

*Corresponding author: telesomonica@gmail.com

Mucocutaneous leishmaniasis is a protozoal disease, transmitted by the bite of Phlebotomus sandflies to mammalian hosts and affects the skin and mucosa. Recent literature have described Leishmania (Viannia) braziliensis $(L V B)$ infections visceralize and affect internal organs such as liver and spleen in hamsters. However, there is a lack of information regarding $L V B$ effects on the reproductive organs. Thus, this study examined the effects of experimental Leishmaniasis caused by $L V B$ on hamster ventral prostate (VP). Briefly, we distributed 12 adult golden male hamsters (Mesocricetus auratus) into two experimental groups: control group (CG) that received only vehicle (saline); and infected group ( $L V B)$ which received $5 \times 10^{6}$ promastigote form of $L V B$ (MHOM/BR/2003/2314) via subcutaneous injection in the right hind paw of the animals. After 15 weeks, the animals were euthanized and VP was dissected and prepared for morphological analysis. There were no alterations in body or glandular weights in controls versus the $L V B$ infected hamsters. The VP from $L V B$ infected animals presented stromal and luminal prostatitis, focal epithelial hyperplasia, alteration in epithelial height and glandular compartments size. In summary, Leishmaniasis infections predisposed the VP to inflammation and promoted changes in glandular architecture. Current reports indicate an increasing incidence of human prostatic diseases such as benign prostatic hyperplasia and prostate cancer without apparent cause. Our results raise the question of a possible secondary origin of these prostatic diseases.

Ethical Committee Protocol CEUA/UEM: 3062060916

\section{Expression of pan-cytokeratin, vimentin and cytokeratin 5 in canine prostatic tissue}

Giulia G. Guiraldelli , Giovana G. Fernandes ${ }^{1}$, Priscila E. Kobayashi ${ }^{1}$, Renée L. Amorim ${ }^{1}$, Carlos Eduardo F. Alves ${ }^{*}$

'São Paulo State University (UNESP), School of Veterinary Medicine and Animal Science, Botucatu, SP.

*Corresponding author: carlos.e.alves@ unesp.br

The origin of canine prostate cancer $(\mathrm{cPC})$ is under discussion and the luminal, basal or ductal cells are the most acceptable origin for $\mathrm{cPC}$. To characterize cPC, we investigated the expression of pan-cytokeratin, vimentin, cytokeratin 5 (CK5). For this, we constructed a tissue microarray, containing 74 canine prostatic tissues samples, all in triplicate. The TMA was composed by 11 normal prostate tissue, 28 benign prostatic hyperplasia (BPH), 16 proliferative inflammatory atrophy (PIA), two prostatic intraepithelial neoplasia (PIN) and $17 \mathrm{cPC}$. An immunohistochemical study was conducted using the mouse monoclonal anti-vimentin (1:300), mouse monoclonal anti-cytokeratin (1:300) and mouse monoclonal anti-cytokeratin 5 (1:50) antibodies. A polymer system was used as secondary antibody and the 3,3'-Diaminobenzidine was used as a chromogen. A descriptive statistical analysis was performed. All canine prostatic samples showed positive pan-cytokeratin expression by epithelial cells. Additionally, five out of 17 cPC had positive cytokeratin cells in the stroma and/or blood vessels. All normal samples and BPH samples presented vimentin positive cells only in stroma. Seven out of 16 PIA samples showed vimentin positive epithelial cells in the lesion foci. Five out of $17 \mathrm{cPC}$ had vimentin positive epithelia cells. All normal and BPH samples showed CK5 expression restricted to the basal cells, presenting a discontinuous basal cell layer. All PIA and PIN samples showed CK5 positive luminal and basal cells. Eight out of $17 \mathrm{cPC}$ presented CK5 positive expression by epithelial cells. Our results indicated a basal-like origin for PIA, PIN and cPC.

\section{Identification of prostate cancer biomarkers in rats submitted to maternal protein restriction}

Ana Carolina L. Camargo ${ }^{*}$, Flávia B. Constantino ${ }^{1}$, Sergio Alexandre A. Santos ${ }^{1}$, Ketlin T. Colombelli ${ }^{1}$, Elian R. David ${ }^{1}$, Luis Antonio Justulin ${ }^{1}$

${ }^{1}$ Institute of Biosciences of Botucatu (IBB) - Sao Paulo State University (UNESP), Botucatu/SP, Brazil.

*Corresponding author: carolgalah@gmail.com 
Recent study shows that maternal protein restriction (MPR) can be linked to prostate carcinogenesis in male rat offspring with aging. However, there is few data on the molecular signaling pathway involved with prostate cancer $(\mathrm{PCa})$ in young offspring. Our objective was to investigate the potential biomarkers involved with $\mathrm{PCa}$ in 21-day-old rat offspring submitted to MPR. Male Sprague Dawley rats were divided into a control group $(\mathrm{CT} \mathrm{n}=4)$, born from mothers fed normal diet $(17 \%$ protein) and GLLP $(\mathrm{n}=4)$, born from mothers fed a low protein diet (6\% protein) during gestation and lactation. The rats were euthanized on postnatal day (PND) 21 and the ventral prostate was processed for proteomic analysis. We analyzed the upregulated proteins in the TargetP, SignalP, SecretomeP and Plasma proteome databases to identify secretion classic/non-classic pathways, localization and presence in plasma. The SurvExpress with PRAD-TCGA was used for validation of biomarkers with cancer gene expression data. Mass spectrometry identified 343 proteins, 78 of which were upregulated and nine were identified as secreted protein (Psbpc1, Calr, Scb2a2, Alb, Hspa5, Klk9, Pdia3, Sbp, Hist1h2ba). In SurvExpress the CALR, HSPA5 and PDIA3 genes, were upregulated in cancer cells and could be related to the potential biomarker for PCa. Thus, our data shown that MPR can alter the expression of molecular pathway during the prostate development, increasing the risk of prostate cancer in adult life.

Financial Support: Fapesp (2017/08715-0; 2017/01063-7), CNPq (142333/2017-4) and Capes.

Ethics Committee on the Use of Animals: 949

\section{Immunoblotting and immunolocalization of the canine- specific arginine esterase using anti-human PSA antibody}

Laíza S. Camargo ${ }^{1 *}$, Priscila E. Kobayashi' ${ }^{1}$, Fabiana F. Souza $^{1}$, Renée L. Amorim ${ }^{1}$, Carlos Eduardo F. Alves'

${ }^{1}$ School of Veterinary Medicine and Animal Science, São Paulo State University. FMVZ, UNESP, Botucatu - Brazil. *Corresponding author: laizacamar@gmail.com

The prostate specific antigen (PSA) is widely used in humans as a marker of prostate cancer (PC), in dogs, a homologous protein called canine-prostate specific arginine esterase (CPSE) has been described. This study aimed to evaluate the immunostaining of PSA in canine prostatic normal tissue and lesions. Samples of seminal plasma were evaluated by Western blot (WB) and the prostate tissue microarray (TMA) for immunohistochemical. For WB, semen was collected from 2 Beagle dogs. After SDS-PAGE, proteins were transferred to a membrane and treated with a primary antihuman PSA antibody (1:1,000, Biorbyt, Cambridge, UK). For the TMA slides, 96-dog prostate tissue samples were used. TMA slides were deparaffinized and antigen retrieval was performed with $\mathrm{pH} 6.0$ citrate buffer. A rabbit antiPSA primary antibody was applied at 1:800 (Biorbyt, Cambridge, UK). A Polymer system was applied as a secondary antibody (Envision, Dako, Carpinteria, CA, USA) and the reaction was developed with 3,3'diaminobenzidine (DAB, Dako, Carpinteria, CA, USA). Results for TMA were presented as positive or negative staining. In the WB, we found one specific band with a molecular weight of $15 \mathrm{kDa}$. Regarding the immunohistochemistry, 6 normal tissues, 28 benign prostatic hyperplasia, two prostatic intraepithelial neoplasia, 16 proliferative inflammatory atrophy and 17 PC were positive on luminal secretory cells. 5 out of 28 PC samples showed positive neoplastic cells invading stroma and vessels. We concluded that the human anti-PSA antibody recognizes the canine CPSE protein and can be used as diagnostic tool in canine prostatic diseases.

\section{Investigation of VEGF and VEGFR expression in canine prostatic tissue}

Antonio Fernando L. Filho ${ }^{1}$, Priscila E. Kobayashi ${ }^{1}$, Renée L. Amorim ${ }^{1}$, Carlos Eduardo F. Alves ${ }^{1 *}$

${ }^{1}$ School of Veterinary Medicine and Animal Science, São Paulo State University. FMVZ, UNESP, Botucatu - Brazil. *Corresponding author: carlos.e.alves@unesp.br

This study aimed to evaluate VEGF and VEGFR protein and gene expression in canine prostatic tissue. We selected 12 normal prostate samples, 12 proliferative inflammatory atrophy (PIA) lesions and 18 canine prostatic carcinomas (PCs). The protein expression was evaluated by immunohistochemistry and gene expression using RT-qPCR. Immunohistochemical staining for VEGF and VEGFR was quantified using manual and computerassisted methods. The gene expression was evaluated using t-test. The survival curve was calculated only for PCs using the Kaplan-Meier method, and the statistical significance was determined using a log-rank test. For statistical propose, we calculated the mean of expression and samples over than mean was called as VEGF or VEGFR high and samples showing expression lower than mean was called as VEGF or VEGFR low. The normal and PIA samples showed negative to weakly positive VEGF and VEGFR expression. The PC samples had VEGF $(\mathrm{P}<0.0001)$ and VEGFR $(\mathrm{P}<0.0001)$ protein overexpression compared to normal samples. We did not find statistical difference between VEGF and VEGFR gene expression among normal, PIA and PC samples. However, we found a positive correlation between VEGF and VGFR expression in normal samples (Spearman $\mathrm{r}=0.68$ and $\mathrm{P}=$ $0.13)$. We also found a positive correlation between VEGF and VEGFR protein expression in normal samples (Spearman $\mathrm{r}=0.8$ and $\mathrm{P}<0.0001$ ). There was no correlation 
of gene or protein expression between VEGF and VEGFR in PC samples. Additionally, the patients with lower VEGFR expression experience a higher survival time.

\section{Low-dose Malathion impairs androgen signaling and prostate morphologyin juvenile rats}

Julia C. Mori ${ }^{*}$, Rafaela P. Erthal ${ }^{2}$, Glaura S. Alves Fernandes $^{3}$, Jaqueline C. Rinaldi ${ }^{4}$

${ }^{1}$ Undergraduated, State University of Maringá DCM/UEM.

${ }^{2}$ MS, Londrina State University - UEL.

${ }^{3} \mathrm{PhD}$, Londrina State University - UEL.

${ }^{4} \mathrm{PhD}$, State University of Maringá - DCM/UEM.

*Corresponding author: juliacmori@gmail.com

Malathion is an organophosphate used to protect public health from diseases like malaria, dengue and others by controlling vectors such as mosquitoes. Human exposures to Malathion can occur via oral, inhalation or dermal routes and its toxicity has been studied in various body organs/systems, including the reproductive system. Review of the literature suggests organophosphate has a direct toxic impact on the Leydig cells. However, there is a lack of information regarding Malathion's effect on prostate development. In this study, our aim was to evaluate the effects of this pesticide on the developing ventral prostate gland (VP) in Wistar rats. Briefly, we distributed 15 male rats into three experimental groups: control group (CG) that received only vehicle (saline); and the treated groups that received Malathion at doses of $10 \mathrm{mg} / \mathrm{kg}$ (GM10) or $50 \mathrm{mg} / \mathrm{kg}$ (GM50) via gavage for 40 days. The animals were euthanized on postnatal day (PND) 65. In comparison to the control rats, there were no alterations in body and glandular weights at PND65. Exposure to Malathion suppressed the levels of testosterone plasma. Histopathology of the VP showed that Malathion suppressed epithelial androgen receptor expression, altered the size of glandular compartments and increased collagen deposition. The prostate is an androgen dependent gland, such that low dose Malathion exposure during the critical development window (puberty) can modify the normal functions of the endocrine system by androgen deprivation. In summary, our results showin the developing VP through puberty low dose Malathion impairs androgen signaling and affectsVP morphology.

Ethical Committee Protocol CEUA/UEL: 12305.2016.65

\section{Maternal protein malnutrition causes hormonal imbalance and leads to the development of prostate cancer}

Ketlin T. Colombelli ${ }^{1 *}$, Luiz Marcos F. Portela ${ }^{1}$, Sérgio Alexandre A. Santos ${ }^{1}$, Ana Carolina L. Camargo ${ }^{1}$, Flávia B. Constantino ${ }^{1}$, Luis Antonio Justulin ${ }^{1}$

${ }^{1}$ Institute of Biosciences of Botucatu (IBB) - Sao Paulo State University (UNESP), Botucatu/SP, Brazil.

*Corresponding author: ketlin.colombelli@gmail.com

Fetal Programming (FP) is adverse gestational conditions that can result in morphological and metabolic alterations in the embryo/fetus during development. Maternal protein malnutrition (MPM) during perinatal period is the most used model to study FP, which already showed that impairs the development, maturation and function of prostate pups. The aim was investigated the impact of MPM on rat ventral prostate during aging. Sprague Dawley male rats $(\mathrm{n}=15 /$ group) whose mothers were fed with control diet (CTR, 23\% protein) or low protein diet (6\% protein) during the gestational (gestational low protein - GLP) or during gestational and lactation period (gestational and lactation low protein - GLLP). At post-natal day (PND) 540, the ventral prostate lobes (PV) and the blood were collected for morphological and hormonal analysis. Both IGF-1 and estrogen were increased in both LPD groups, while testosterone was decreased. The incidence and severity of prostate lesions were increased in LPD groups. It was observed: epithelial atrophy, physiological and focal hyperplasia, epithelial dysplasia, intraepithelial neoplasia and carcinoma in situ. The most voluminous carcinoma in situ, an intense microangiogenesis was observed. The estrogen/androgen ratio is higher in both LPD groups compared to CTR. The increased estrogen/testosterone ratio in LPD offspring could be related to the prostatic disorders. Although the estrogenic levels normally increase during perinatal period, it declines during post-natal life. The altered intrauterine microenvironment appears to program offspring prostate cells, which respond to the imbalance of hormones in older rats, leading to a slow-growing prostatic carcinogenesis.

Ethics Committee on the Use of Animals: CEUA 951/2017- IBB/UNESP

Financial Support: Fapesp (2017/01063-7 and 2017/14690$0)$ and Capes.

Proliferative inflammatory atrophy and carcinomas from canine prostate are enriched of intermediate cells

Giovana G. Fernandes ${ }^{1}$, Giulia G. Guiraldelli ${ }^{1}$ Priscila E. Kobayashi $^{1}$, Renée L. Amorim ${ }^{1}$, Carlos Eduardo F. Alves ${ }^{1 *}$

${ }^{1}$ São Paulo State University (UNESP), School of Veterinary Medicine and Animal Science, Botucatu, SP. *Corresponding author: carlos.e.alves@unesp.br 
Proliferative inflammatory atrophy (PIA) is a preneoplastic lesion, widely described in human medicine. Previous studies had shown an intermediate phenotype (basal marker ${ }^{+} /$luminal marker ${ }^{+}$) in PIA lesions in humans. Thus, our research aimed to identify an intermediate cells phenotype in proliferative and neoplastic lesions of the canine prostate. We have collected 74 prostatic tissues from intact dogs aging 1 to 15 years-old and diagnosed 11 normal samples, 28 benign prostatic hyperplasia (BPH), 16 PIA, two prostatic intraepithelial neoplasia (PIN) and 17 prostate cancer (PC). We constructed a tissue microarray (TMA) slide with these group of samples, using the Tissue Microarrayer (Beencher Instruments, Silver Spring, USA), with all samples in triplicate. Double immunohistochemistry technique, for p63 (Brown color, DAB, Dako cytomation, Carpinteria, CA, USA) and CK8/18 (Red color, AEC, Dako cytomation, Carpinteria, CA, USA) was performed in the TMA slide. A descriptive statistical analysis was performed. All normal (11/11) and BPH (28/28) samples had no double stained cells. The luminal cells were positive for cytokeratin (red staining) and negative for $\mathrm{p} 63$ (brown staining). On the other hand, basal cells were negative for cytokeratin $8 / 18$ and positive for p63. All PIA (16/16), PIN (2/2) and samples showed doubled stained cells $\left(\mathrm{CK} 8 / 18^{+} / \mathrm{P}^{+} 3^{+}\right)$. Regarding the PC, eight out of 17 samples had doubled stained cells $\left(\mathrm{CK} 8 / 18^{+} / \mathrm{P} 63^{+}\right)$. The remaining 12 samples, showed positive CK8/18 staining only in luminal cells and p63 positive staining restrict to basal cells. Our results indicates that PIA and some canine PC are enriched of intermediate cells $\left(\mathrm{CK} 8 / 18^{+} / \mathrm{P} 3^{+}\right)$.

\section{Toxoplasmosis impairs Ventral Prostate Morphophysiology}

Hiohana R. M. Sacon ${ }^{1 *}$, Suellen L. V. Vieira ${ }^{2}$, Debora M. G. Sant'Ana ${ }^{2}$, Sérgio Luis Felisbino ${ }^{3}$, Jaqueline C. Rinaldi ${ }^{2,3}$

${ }^{1}$ Undergraduated.

${ }^{2} \mathrm{PhD}$ - State University of Maringá - UEM, Maringá/PR. ${ }^{3} \mathrm{PhD}$ - São Paulo State University - UNESP, Botucatu/SP. *Corresponding author: hiohanarilary@gmail.com

Toxoplasmosis is a zoonosis caused by the apicomplexan parasite Toxoplasma gondii. The parasite generally enters the body via the oral route, which occurs through the ingestion of oocysts or cysts present in contaminated water and food. Review of the literature suggests toxoplasmosis promote important reproductive problems in females such as changes in the estrous cycle, miscarriages, birth of preterm animals or even fetal malformations. However, the reproductive consequences in infected males have been neglected. Thus, we questioned whether $T$. gondii could impair rat ventral prostate (VP) morphophysiology. Briefly, we distributed
18 Wistar male rats into three experimental groups: control group (CG) that received only vehicle (saline); and the treated groups that received a unique dose of 500 (G500) or 5.000 (G5.000) oocysts of $T$. gondii (strain ME-49, genotype II) via gavage. After 30 days, the animals were euthanized (on postnatal day 70), the VP was dissected and prepared for morphological and biochemical analysis. In comparison to the control rats, the infected animals did not show alteration in body or glandular weights. The main changes found in the VP of G500 and G5.000 were a decrease in epithelial height, an increase in stromal area and an increased incidence of focal epithelial hyperplasia and prostatitis. No changes in androgen receptor expression were observed, however an imbalance of PCNA/PAR-4 expression occurred. In summary, the toxoplasmosis predisposed the prostatic tissue to inflammation and promoted changes in glandular architecture.

Ethical Committee Protocol CEUA/UEM: 081/2012 